\title{
Scalar vs. Epistemic Vagueness: Evidence from Approximators
}

\author{
Uli Sauerland and Penka Stateva \\ Zentrum für allgemeine Sprachwissenschaft
}

\section{Introduction}

This paper is about vagueness in natural language. More specifically, we discuss lexical means of making vague assertions more or less precise. Examples of expressions that have this effect are approximately, absolutely, definitely, and roughly speaking. While many of these expressions are modifiers and adverbs, some such expressions are neither. Hence, for the purposes of this paper we call expressions that make vague assertions more or less precise Approximators. Our main claim is that the distribution of such expressions provides evidence for the view that vagueness in language comes in at least two varieties, which we call Scalar Vagueness and Epistemic Vagueness. ${ }^{1}$

We assume that vagueness is characterized by giving rise to the SoritesParadox. Consider the three examples in (1) through (3). In each case a SoritesParadox reasoning goes through: If we accept the premise that "If Harry is bald, it's always true that, even if he had one more hair than he does, he would still be bald.", which sounds innocent, it follows that (1) does not tell us anything about the number of hairs on Harry's head. Similarly (2) would tell us how many grains of sand there are in the heap if we accept the premise that "if something is a heap of sand and we remove one grain from it, it is still a heap of sand.". Finally, (3) wouldn't tell us anything about the time of John's arrival if we accept the premise that "if John arrived at 6 o'clock, then (3) would still be true if he arrived one second later than he actually did."

\section{(1) Harry is bald.}

(2) This is a heap of sand.

(3) John arrived at 6 o'clock.

Earlier versions of this paper were presented at Semantiknetzwerk 6 at University Pompeu Fabra in Barcelona, the European Conference on Philosophy and Psychology at Queens University in Belfast, the ZAS Semantikkreis in Berlin, and the Tokyo Area Semantics Circle at the National Institute for Informatics in Tokyo. In addition to these audiences, we thank Manfred Krifka, Manfred Bierwisch, Graham Katz, Louise McNally, Chris Tancredi, Sigrid Beck, Anke Lüdeling, Amir Zeldes and Philippa Cook for their input. We thankfully acknowledge the financial support from the German Research Council DFG (grants SA 925/1 in the Emmy-Noether Program and SA 925/2 to U. Sauerland). Of course, we take full responsibility for any remaining errors.

${ }^{1}$ The term imprecision in work by Pinkal (1995), Kennedy (2007) partially overlaps with what we refer to as scalar vagueness, but not completely so. For example, bald is usually regarded as vague rather than imprecise. Hence, we introduce two new terms in this paper. Our terminology also reflects that we regard both phenomena as a kind of vagueness. 
We contrast two views of vagueness: the monistic and the dualistic view. The monistic view of vagueness, on the one hand, assumes that there is one general mechanism of vagueness that underlies all phenomena exhibiting vagueness in natural langauge. Adherents to the uniform view do not all agree on what the general mechanism of vagueness is, but they agree that there is just one mechanism. For instance, the following treatments of vagueness advocate a uniform view: Lakoff (1973), who analyses all forms of vagueness using fuzzy logic, Kamp (1981), who uses supervaluations, Lasersohn (1999), who proposes "Pragmatic Halos" for all forms of vagueness, and to some extent also the epistemic view of vagueness of Williamson (1994). The dualistic view, on the other hand, assumes that there are at least two mechanisms that can give rise to vagueness. Adherents to the dualistic view like Pinkal (1995), and Kennedy (2007) have, as far as we are aware of, generally distinguished between (2) and (3). Intuitively, the distinction is that there seems to be no precise concept of heap, while there is a precise concept of 6 o'clock, a point in time. However, the expression 6 o'clock does not refer to this precise concept, but rather a broader, vague one. This intuitive distinction by itself, however, would not require two different accounts of vagueness in language. Furthermore, there are cases where the intuitive distinction is drawn in varied ways: Existing work that advocates the dualistic view does not say where expressions like bald in (1) are classified. Bald is generally considered a core case of vagueness in language and is often used to exemplify the concept (for instance, by Kamp 1981 and Williamson 2000: 102), but it also is intuitively related to a precise conceptthat of having no hair whatsoever on the scalp. The evidence from approximators we discuss in this paper not only provides new evidence for the dualistic view, but also gives us a way of classifying vague expressions into one type or another. Specifically, we argue below that bald exhibits scalar vagueness.

\section{Initial Support for the Dualistic Theory: Distributional Differences Between Approximators}

The example in (4) illustrates that approximators have a limited distribution: while exactly and approximately easily combine with fifty, they are unacceptable with heap, as in (4b):

(4) a. What John cooked was exactly/approximately fifty tapas.

b. \#What John cooked was exactly/approximately Beef Stroganoff.

The contrast in (4) between different vague expressions is unexpected from the point of view of the monist theory of vagueness. Specifically, this point is shown below for the analysis of Lasersohn (1999), which is the only monist analysis we are aware of that provides an account of some approximators. On our dualist account, however, the difference between five and Beef Stroganoff follows from that between scalar and epistemic vagueness. 
There are also differences between approximators. Consider for example, the expressions definitely and maybe. They can be used to express approximation: For example, (5) when used while we are eating the dish John cooked. ${ }^{2}$

What John cooked is definitely/maybe Beef Stroganoff.

We take these distributional differences to suggest that there are two groups of approximators: scalar approximators and epistemic approximators. The two classes correspond to the two kinds of vagueness, scalar and epistemic vagueness. ${ }^{3}$

There are several other approximators that we consider in detail below. Specifically, we further distinguish between two kinds of approximators in the scalar domain: For instance, absolutely, completely, and totally are more or less in complementary distribution with exactly. We show below that exactly is used when the associated scalar expression denotes a mid-point of a scale while absolutely, completely, and totally are used only with endpoints (Kennedy and McNally 2005).

(6) a. *What John cooked was absolutely/completely/totally fifty tapas.

b. What John cooked was absolutely/completely/totally appropriate.

For the English examples, it is necessary to mention on further property of exactly right away. In the scope of negation exactly can combine with any kind of predicate and in that respect patterns with the epistemic approximators.

Red wine isn't exactly healthy.

This seems to cast doubt on the validity of the argument for distinguishing among kinds of vagueness. But, there are two reasons to believe that (7) does not threaten the suggested classification. For one, consider the German and Bulgarian counterparts of exactly. In (8a) and (9a) these are literal translations of English exactly. But, unlike English exactly, both Bulgarian točno in (8b) and German genau in (9b) are not acceptable with epistemically vague predicates in negative contexts.
a. červenoto vino e točno trigodisno.
red-the wine is exactly three-year-old
b. \#červenoto vino ne e točno zdravoslovno red-the wine is not exactly healthy

\footnotetext{
${ }^{2}$ In such contexts, it is the rather the meaning of the phrase Beef Stroganoff that is discussed, rather than the ingredients and method of preparation of the dish in front of us (Barker 2002).

${ }^{3}$ Epistemic approximators can also be used with expressions that are scalar vague as in (i). A purely approximating interpretation of (i) is available, though only in scenarios like the following: John volunteered to cook fifty tapas. He cooked a lot, but it were exactly fourtynine tapas. Now we are discussing whether John honored the contract. If I believe that John's orginal utterance 'I will make fifty tapas' implied only that we would make about fifty, I could use (i) to state my believe. Note though that this meaning is still quite different from exactly fifty.
}

(i) The number of tapas John cooked is definitely fifty. 
(9) a. Der Rotwein ist genau drei Jahre alt.

the red-wine is exactly three years old

b. \#Der Rotwein is nicht genau gesund.

the red-wine is not exactly healthy

In addition, even precisely, which is a near-equivalent of exactly in English, does not pattern with it:

(10) \#Red wine isn't precisely healthy.

We conclude then that English has a second lexical entry for exactly, which is a strong negative polarity item and an epistemic approximator, and leave it aside in the following.

A further case, where a scalar approximator can be combined with an epistemically vague expressions is that of not even approximately healthy as in (11). These examples can be translated literally into German and Bulgarian, and hence we have no evidence that we are looking at a separate lexical entry for approximately in (11). Nevertheless, this ambiguity analysis presently still seems to be the simplest analysis for (11) to us.

(11) a. Red wine isn't even approximately healthy.

b. What John cooked isn't even approximately Beef Stroganoff.

\section{Proposal}

The dualistic theory of vagueness that we advocate is based on the observation that markers of approximation come in two classes depending on the kind of vagueness they make more precise or more vague. Vague predicates, we suggest, should be described as scalarly or epistemically vague.

\subsection{Scalar Vagueness}

The first kind of vagueness can only be related to expressions that denote a point on a scale. Numerals can serve as a prototypical example. We have already mentioned in Section 1 above that numerals allow for an interpretation that is consistent with a larger segment of the scale: they can denote an interval (Krifka 2007). For example, the expression 5 meters could, in a given context, be a good description of the length of a rod the actual length of which we believe to lie somewhere in the interval between $4.5 \mathrm{~m}$ and $5.5 \mathrm{~m}$. In this example, the scale is partitioned in segments of $1 \mathrm{~m}$.

Following Krifka (2007), we assume the model for the alignment of pointdenoting scalar terms with granularity intervals of a scale that is illustrated by figure 1. A scale can be simultaneously divided up into intervals of varying granularity. In principle, each point-denoting term is a candidate for denoting any intervals that 


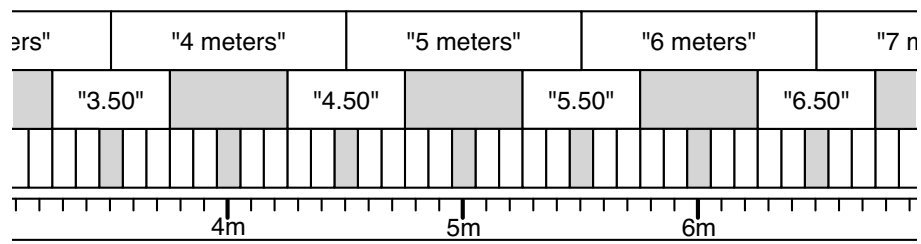

Figure 1: Alignment of length terms with scale intervals

contains this point. However, general pragmatic principles entail that the shortest expression must be used for each interval. ${ }^{4}$ This yields the alignment of terms and scale intervals shown in figure 1 . For instance, 5 meters denotes the interval from 4.50 to 5.50 at the $1 \mathrm{~m}$ granularity, while 4 meter 50 and 4 meter 90 are blocked from denoting this interval because they are longer expressions than 5 meters. Instead, 4 meter 50 denotes an interval at the half-meter granularity, and 4 meter 90 at the $10 \mathrm{~cm}$ interval granularity. The grey intervals in figure 1 are not denoted by any expression of the form $x$ meters $y$ because the shortest expressions denoting a point in these intervals already denote a larger interval.

\subsection{Granularity Functions}

We propose that granularity is a contextual parameter of interpretation. Formally, we assume that a granularity function maps each point of a scale to an interval that contains it. Here are examples of different extensions of 5 meters which vary because of a different setting of scale granularity:

$$
\begin{array}{ll}
\text { a. } & \operatorname{gran}_{\text {fine }}(5 \mathrm{~m})=[4.95 \mathrm{~m}, \ldots, 5.00 \mathrm{~m}, \ldots, 5.05 \mathrm{~m}] \\
\text { b. } & \operatorname{gran}_{\text {mid }}(5 \mathrm{~m})=[4.75 \mathrm{~m}, \ldots, \ldots, 5.00 \mathrm{~m}, \ldots, \ldots, 5.25 \mathrm{~m}] \\
\text { c. } & \operatorname{gran}_{\text {coarse }}(5 \mathrm{~m})=[4.50 \mathrm{~m}, \ldots, \ldots, \ldots, 5.00, \ldots, \ldots, \ldots, 5.50 \mathrm{~m}]
\end{array}
$$

Normally, several granularities are under consideration simultaneously, and hence the gran parameter of interpretation contains more than one granularity function. When a scalar point expression is evaluated, it is mapped by the coarsest granularity such that the expression is the shortest expression that could denote the resulting interval. Hence, we end up with the mapping in (13).

$$
\begin{array}{ll}
\text { a. } & \llbracket 5 \text { meters } \rrbracket^{\text {gran }}=\operatorname{gran}_{\text {coarse }}(5 \mathrm{~m})=[4.50 \mathrm{~m}, 5.50 \mathrm{~m}] \\
\text { b. } & \llbracket 4 \text { meters } 50 \rrbracket^{\text {gran }}=\operatorname{gran}_{\text {mid }}(4.5 \mathrm{~m})=[4.25 \mathrm{~m}, 4.75 \mathrm{~m}] \\
\text { c. } & \llbracket 4 \text { meters } 90 \rrbracket^{\text {gran }}=\operatorname{gran}_{\text {finest }}(4.9 \mathrm{~m})=[4.85 \mathrm{~m}, 4.95 \mathrm{~m}]
\end{array}
$$

Our approach also applies to non-numeral expressions like the middle. We assume that, if $\mathrm{A}$ is actually the center of the circle, the denotations of the middle for different granularity functions could be the following:

\footnotetext{
${ }^{4}$ Krifka shows that in some cases other considerations of cognitive efficiency override the use of the shortest expressions. For example, 18 months denotes a bigger interval when describing a childs age than twenty months.
} 
(14) 【the middle of the circle around $\mathrm{A} \rrbracket$

a. $\operatorname{gran}_{\text {finest }}(\mathrm{S})(\mathrm{A})=$ the $1 \mathrm{~mm}$ circle surrounding $\mathrm{A}$

b. $\operatorname{gran}_{\text {med }}(S)(A)=$ the $1 \mathrm{~cm}$ circle surrounding $A$

c. $\operatorname{gran}_{\text {coarse }}(S)(A)=$ the $2 \mathrm{~cm}$ circle surrounding $A$

Our principles need to be generalized to many other scales. We assume for closed scales the following definitions for granularity function and the notions finer/coarser: A granularity function $\gamma$ for scale $S$ has the following properties:

$$
\begin{array}{ll}
\text { a. } & \forall s \in S: s \in \gamma(s) \\
\text { b. } & \forall s \in S: \gamma(s) \text { is convex } \\
\text { c. } & \forall s, s^{\prime} \in S: \max (\gamma(s))-\min (\gamma(s))=\max \left(\gamma\left(s^{\prime}\right)\right)-\min \left(\gamma\left(s^{\prime}\right)\right)
\end{array}
$$

A granularity function $\gamma$ is finer (or coarser when $<$ is replaced by $>$ ) than $\gamma^{\prime}$ if:

$$
\forall s \in S: \max (\gamma(s))-\min (\gamma(s))<\max \left(\gamma^{\prime}(s)\right)-\min \left(\gamma^{\prime}(s)\right)
$$

These definitions will be sufficient for our purposes in this paper. Ultimately, though, it would be desirable to extend the approach to open scales, which Hobbs and Kreinovich (2006) argue have logarithmic granularity.

\subsection{Scalar Approximators}

Under this view, what is the role of scalar approximators? Let us go back to an example from the introduction (repeated from 4):

What John cooked was approximately/exactly fifty tapas.

We propose that scalar approximators reset the granularity parameter to the coarsest granularity. For this reset, the following new composition principle is needed (assuming the general framework of composition of Heim and Kratzer 1998):

(18) Granularity modifying composition: If $\llbracket \mathrm{A} \rrbracket^{\Gamma}$ has in its domain functions that take sets of granularity functions as arguments, then the new composition rule $\llbracket \mathrm{A} \mathrm{B} \rrbracket^{\Gamma}=\llbracket \mathrm{A} \rrbracket^{\Gamma}\left(\lambda \Gamma^{\prime} \llbracket \mathrm{B} \rrbracket^{\Gamma^{\prime}}\right)$ must be applied.

The lexical entries for approximately and exactly can then be given as in (19). Both set the granularity parameter for the evaluation of their complement to a singleton set; exactly to the finest and approximately to the coarsest granularity.
a. $\quad \llbracket$ exactly $\rrbracket^{\text {gran }}(\mathrm{G})=\mathrm{G}(\{$ finest $($ gran $)\})$
b. $\quad$ aapproximately $\rrbracket^{\operatorname{gran}}(\mathrm{G})=\mathrm{G}(\{\operatorname{coarsest}($ gran $)\})$ 
This approach predicts that the use of exactly makes it possible to denote some of the grey intervals in figure 1. For instance, exactly five meters denotes the interval from $4.95 \mathrm{~m}$ to $5.05 \mathrm{~m}$ in a context where exactly the three granularities drawn in 1 are under consideration.

Our account also predicts the oddity of (20) in a general context: On the coarsest scale, 49 and 50 belong to the same interval. But then, 50 must be used over 49 to denote this interval.

\#What John cooked was approximately 49 tapas.

We give lexical entries for other scalar approximators in Section 4 below, and also discuss the restriction to midpoints of exactly and approximately. We argue there that at least the following expressions belong to this class:

(21) a. Scalar more precise approximators: Exactly, absolutely, completely, precisely, perfectly

b. Scalar less precise approximators: approximately, about, partially, sufficiently, roughly.

\subsection{Epistemic Vagueness and Approximators}

We observed above that expressions like definitely and maybe can be used as approximators. In contrast to scalar approximators, they can combine with any predicate that does not have a precise meaning or at least it is not known. For such predicates we use the term epistemically vague (cf. Bosch 1983, Williamson 1994). We propose that epistemically vague predicates differ in their extensions even across worlds where physical object properties (i.e. the number of sand grains in a heap) do not differ. Let us take heap as a prototypical example of an epistemically vague predicate. As illustrated in (22), we assume that the minimum amount of sand that constitutes a heap can differ: the extension of heap may include in a possible world $w_{1}$ any pile of more than 20 grains, but in a possible world $w_{2}$ it may include only objects consisting of more than 30 grains even though the two worlds are indistinguishable in terms of the location and size of objects.

$$
\begin{aligned}
& \text { heap }\left(w_{1}\right)=\langle\text { twenty grains, twenty-one grains, } \ldots\rangle, \\
& \text { heap }\left(w_{1}\right)=\langle\text { thirty grains, thirty one grains, } \ldots\rangle
\end{aligned}
$$

This approach predicts that epistemic approximation arises as a side effect of general epistemic quantification. Since the epistemic approximators also all have general epistemic uses, this is a desirable result. Approximation is most clearly intended when there is no other uncertainty: ${ }^{5}$

\footnotetext{
${ }^{5}$ Makoto Kanazawa (p.c.) points out that also example (i) brings about an approximation interpretation and that furthermore there is an interesting contrast between (i) and (ii). The contrast is explained if a felicitous use of the heap requires that in all worlds of the common ground there must be a salient referent for it, and furthermore presupposes that they are satisfied if evaluated against
} 
(23) This perfectly cone-shaped pile of 17 sand-grains on the table in front of us is maybe/definitely a heap.

We hence assume that maybe and definitely express existential and universal epistemic quantification respectively and that the effect of approximation is a consequence of this use. We think that this approach should also be extended to other epistemic approximators, such as the following: ${ }^{6}$

(24) a. Epistemic more certain approximators: definitely, positively, for sure, certainly

b. Epistemic less certain approximators: more or less, maybe, -ish

English epistemic modals also can be used as approximators, but since they have an evidential component requiring indirect evidence (von Fintel and Gillies 2006), this requires a special context. For example, (25) could be used by me in a context where I just heard a third person, who speaks English natively, refer to the sand-accumulation in front of me as a heap:

This cone-shaped pile of 17 sand-grains must be a heap.

\subsection{Combinatorics: Further Support for the Dualistic View}

Stacking approximators provides further support for the dualistic view. Our theory predicts that scalar approximators could not be stacked for the following reasons: Recall we suggested that these approximators restrict the granularity parameter of their complement to one granularity function. A second scalar approximator in the scope of the first is vacuous. The facts, as we see in (26) and (26), coincide with the predictions.

(26) a. \#John is exactly/precisely approximately 30.

b. \#John is approximately exactly/precisely 30 .

the common ground.

(i) This heap is definitely a heap.

(ii) \#This heap is maybe not a heap.

${ }^{6}$ Gillian Ramchand (p.c.) points out that in some dialects of British English ish can be used not just as an affix as in greyish or Beef Stroganoffish, but also as a independent sentence final morpheme. A Linguist List post by Margaret Fleck from February 1992 points out the following example (http://linguistlist.org/issues/3/3-129.html):

(i) A: Is your algorithm working?

B: Yes, it's working. Ish.

The affix -erly seems restricted to compass direction as in northerly vs. northern. Judging from the data reported in Siegel (2002), English like behaves also like an epistemic approximator in that it is not restricted to scalar predicates. Geoff Pullum, in a November 22, 2003 Language Log internet post, points out that if you will is similar in distribution and meaning to like (http://itre.cis.upenn.edu/\%7Emyl/languagelog/archives/000138.html). 
Winter (2004) and Kennedy and McNally (2005) argue that the scales associated with adjectives can be either open or closed, and that this affects the denotation of adjectives. Specifically, adjectives associated with closed scales denote the endpoints of the scale, while those associated with open scales cannot denote endpoints. Hence, our proposal predicts, on the one hand, that the endpoint-associated approximators should combine only with the closed scale adjectives. Following Kennedy and McNally (2005), we explain the distribution of absolutely in (31) on this basis. Approximators like exactly, which are not restricted to endpoints, on the other hand, can also only combine with point-denoting scalar expressions. The semantics we gave in (19) above for exactly initially predicts that it can combine with any point-denoting scalar expression. But, we expect furthermore that exactly should be blocked whenever a more specialized expression such as absolutely is applicable. Therefore exactly is expected to combine only with expressions that denote non-endpoints on a scale. In this way, we explain the distribution of exactly in (31).

a. The glass is absolutely/\#exactly full.

b. The glass is exactly/\#absolutely half full.

Intuitions on the use of approximators are sometimes fleeting. For instance, (31a) with exactly improves after pondering it for a while. We think this is due to some coercion of the scale structure where full does not denote an endpoint. This is supported by an intuition that Chris Tancredi (p.c.) pointed out to us: exactly full could be used to describe a glass in which the liquid is exactly level and aligned with the upper rim of the glass; absolutely full though could be used to describe a glass where the surface of the liquid it contains is bulging upwards higher than the brim of the glass.

To circumvent the effect of scalar coercion, we also tested our distributional claims with a corpus study using the British National Corpus (BNC). The result of this study is reported in figure 2 . We measured how frequent an approximatoradjective sequence occurs relative to the individual frequency of approximator and adjective. The numbers we report in (32) are such that if approximator and adjective were randomly distributed in the corpus, the value 1 should appear. ${ }^{8}$ The columns of the table each show for each approximator that number of individual occurrences in the corpus altogether in the second row. Beginning from the third row, a relative frequency scores is shown for specific scalar expressions. This value is calculated according to the formular at the bottom of the diagram: the number of occurrences of the string of approximator followed by the scalar expression divided by the number of individual occurrences of each word. The rows contain for each of the adjectives we looked at, the number of individual occurrences in the first column and the frequency scores for each approximator in the following columns.

\footnotetext{
${ }^{8}$ Technically, the value reported for a random distribution should be close to 1 , but not necessarily exactly 1 .
} 


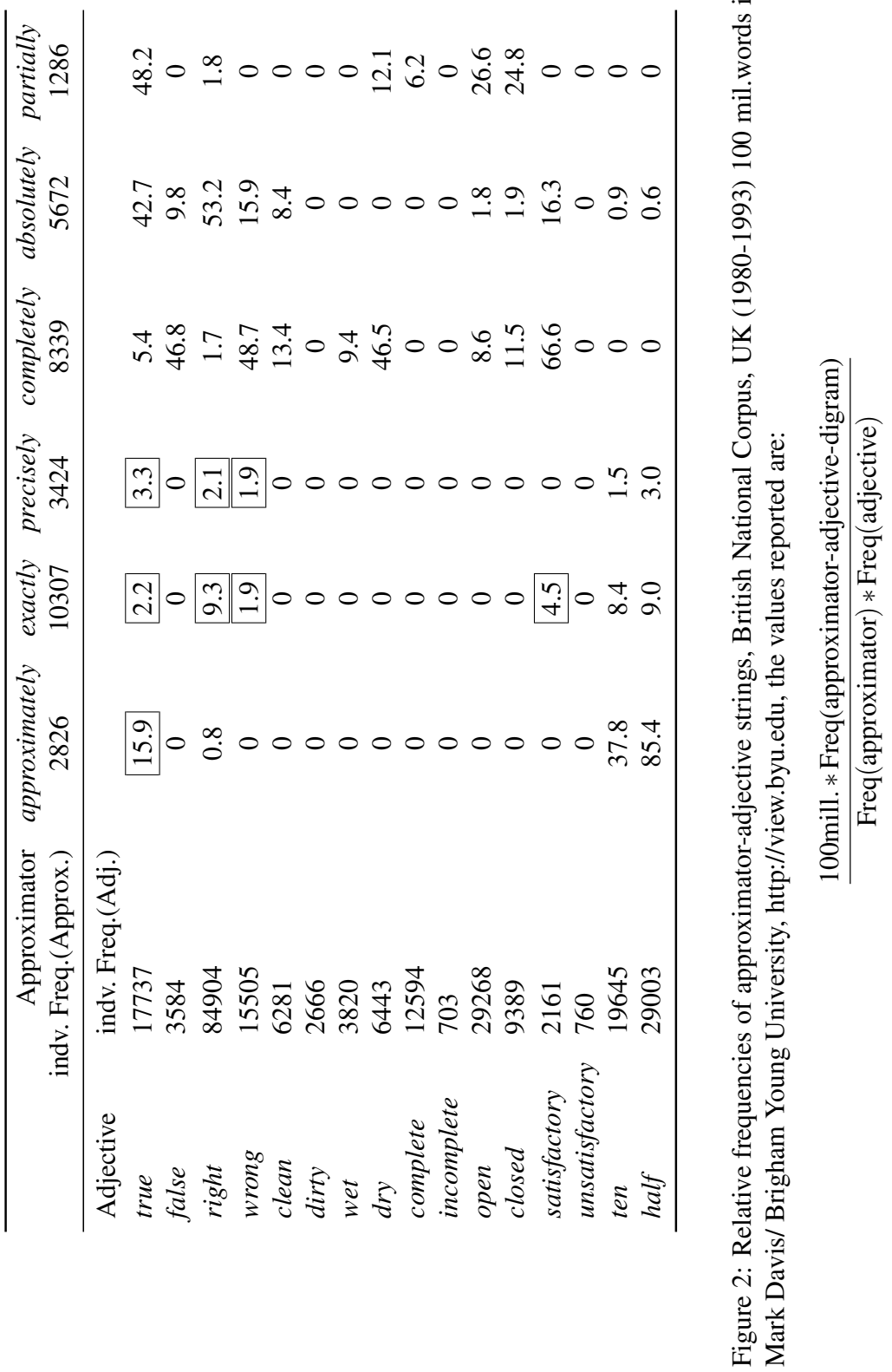


The results for the most part confirm our expectations. We have marked the unexpectedly high values with a surrounding line. We did not mark unexpected 0 values since we assume that the corpus is too small to conclude anything from these. The three most notable departures from our predictions are the frequencies of approximately true, exactly right, and exactly satisfactory. We assume that each of these is explained by reference to a coerced scale structure, but two different processes of coercion are at work. For exactly satisfactory, we think that a scale where satisfactory denotes a non-endpoint between unsatisfactory and great is available. For right and true, we assume that the scale of truth can be adjusted to have truth as a midpoint between two poles of falsity as shown in (32).

$$
\text { false true false }
$$

We assume that the scale in (32) is available when the truth of an nonendpoint answer to a scalar question asking is relevant. Hence we expect the contrast in (33):
a. A: How tall is Chris?
B: $190 \mathrm{~cm}$.
A: You're exactly right./That's approximately true.
b. A: How dry do you think the shirt is?
B: It should be dry by now.
A: \#You are exactly right.

\subsection{More Precise Scalar Approximators}

Before looking at other approximators, recall again the distribution of exactly as shown in (34): It cannot combine with the endpoint denoting scalar expressions in (34a). With the non-endpoint denoting scalar expressions in (34b), however, exactly is fine. These include numerals, time descriptions, spatial boundaries, same, and equatives. Finally, non-NPI exactly cannot combine with the non-scalar expressions in $(34 c)$.
a. \#exactly dry/pure/full/empty/white
b. exactly three/?north/the same
c. \#exactly Beef Stroganoff/a heap of wood

Our lexical entry for exactly in (19) at this point only explains the restriction of exactly to scalar vague terms: because granularity is not made use of by these predicates, exactly is vacuous. The restriction to non-endpoints we assume follows from the fact that there are expressions like absolutely and completely that specifically make the endpoints more precise.

For the semantic entries of further approximators we adopt the convention that $d$ be the type of degrees. We assume furthermore that adjectives are of the type $\langle\langle d, t\rangle,\langle e, t\rangle$. For example, for dry we assume the lexical entry in (35). 
$\llbracket$ dry $\rrbracket^{\mathrm{w}, \text { gran }}=\lambda I \in D_{\langle d, t\rangle} \lambda x \in D_{e} . I$ holds of the degree to which $x$ is dry

Absolutely, completely, and totally are all three in complementary distribution with exactly in the scalar domain. As Kennedy and McNally (2005) have already observed, completely can combine only with predicates which denote endpoints of a scale in (36a). It does not combine with non-endpoints in (36b) or with epistemically vague expressions in (36c). The distribution of absolutely and totally is very similar to that of completely as far as we can tell, and in the following we only talk about completely. ${ }^{9}$
a. completely dry/pure/white
b. \#completely three/north/the same
c. \#completely Beef Stroganoff/a heap of wood

The lexical entry in (37) is similar in spirit to Kennedy and McNally's (2005) suggestion, but it makes reference to the function of the modifiers as granularity setters.

$$
\begin{gathered}
\llbracket \text { completely } \rrbracket^{\text {gran }}=\lambda f \in D_{\langle d t, e t\rangle} \lambda x \in D_{e} \\
\left.\left.\exists D \in D_{d t}[D=\text { finest(gran)(max (domain }(f))\right) \& f(D)(x)\right]
\end{gathered}
$$

This entry takes an adjective as argument and returns a property. Hence, the lexical entry explains why completely must be adjacent to the scalar expression it associates from exactly as shown by (38). Since it makes reference to the endpoint, (37) furthermore explains why completely requires closed scales.

(38) a. Mary arrived (*completely) with (completely) dry shirts.

b. Mary arrived (exactly) at (exactly) noon.

Precisely has a distribution similar to that of exactly, but is preferably combined with anaphoric expressions denoting non-end points of a scale, as illustrated in (39). At present, we assume that precisely has the same lexical entry as exactly in (19).
a. \#precisely dry/pure/full/empty/white
b. ?precisely three/that amount/\#north/there
c. \#precisely Beef Stroganoff/a heap of wood

Perfectly also belongs to this group of scalar approximators, and its meaning and distribution is closely related to that of completely:

(40) a. perfectly dry/straight/clean/safe/appropriate/possible \#perfectly full/empty/white

b. \#perfectly three/north

\footnotetext{
${ }^{9}$ We have to put aside for now, though, uses of absolutely with quantifiers like every and no. Furthermore, absolutely can combine with zero, while completely and totally cannot.
} 


\section{c. \#perfectly Beef Stroganoff/a heap}

Perfectly has, however, an intensional component. Intuitively, an expression like perfectly dry makes reference to a desired point of dryness. ${ }^{10}$ Given that, it should be analyzed in a way similar to modal superlatives like the driest possible. Building on this intuition, we can suggest a lexical entry for perfectly that follows closely, in the relevant respect, Schwarz's (2006) definition of the modal superlative operator -est-possible. We assume that like -est-possible, perfectly moves to take sentential scope. It leaves behind a trace of the type $\langle d, t\rangle$, and combines with the lambdaabstract created by movement. The lexical entry for perfectly in (41) applied to The shirt is perfectly dry can be paraphrased as: The shirt is completely dry and complete dryness of the shirt is desirable.

$$
\begin{gathered}
\llbracket \text { perfectly } \rrbracket^{w, \text { gran }}=\lambda R \in D_{\langle d t, s t\rangle} \forall w^{\prime} \in D_{s} \exists D \in D_{\langle d, t\rangle}\left[\operatorname{Acc}_{w}\left(w^{\prime}\right) \&\right. \\
\left.D=\text { finest(gran)(max(domain }(R))) \& R(D)(w)=1 \& R(D)\left(w^{\prime}\right)=1\right]
\end{gathered}
$$

The similarity in meaning between modal superlatives and perfectly explains the oddity of (42):

\#The shirt is completely driest possible.

\subsection{Less Precise Scalar Approximators}

Approximately clearly patterns with exactly in its distribution. It, too, combines only with scalar expressions not associated with endpoints as shown in (43).
a. \#approximately dry/pure/white
b. approximately three/north/the same
c. \#approximately Beef Stroganoff/a heap of wood

The lexical entry in (19) explains why the use of approximately with epistemically vague expressions is blocked in the same way as for exactly. For the restriction to endpoints, we claim that the use of approximately is blocked by more or less, just as exactly was blocked by completely.

More or less we analyze as the counterpart of completely, making reference to the coarsest granularity that combines with endpoints as shown in (44). ${ }^{11}$
a. more or less dry/pure/white
b. \#more or less three/north
c. \#more or less Beef Stroganoff/a heap of wood

Our lexical entry for more or less is given in (45): ${ }^{12}$

\footnotetext{
${ }^{10}$ Eric McCready (p.c.) points out uses like perfectly awful and perfectly horrible where perfection is not related to desirability. We put these cases aside for now.

${ }^{11}$ One exception to the complementarity of approximately and more or less is more or less the same. We put this aside for now.

${ }^{12} \mathrm{As}$ in the case of absolutely, we restrict ourselves here to uses of more or less with adjectives.
} 


$$
\begin{aligned}
& \llbracket \text { more or less } \rrbracket^{\text {gran }}=\lambda f \in D_{\langle d t, e t\rangle} \lambda x \in D_{e} \\
& \exists D \in D_{d t}[D=\text { coarsest }(\operatorname{gran})(\max (\operatorname{domain}(f))) \& f(D)(x)]
\end{aligned}
$$

Note that more or less must be adjacent to the scalar expression it is construed with again, as is the case with completely.

(46) John arrived (\#more or less) with (more or less) clean clothes.

Pretty has a use that is in many ways similar to more or less, but we still need to investigate it in more detail.

About is similar to approximately but can only be construed with numerals and temporal expressions as shown in (47).

a. about three, at about noon, at about midnight, at about the same time

b. \#about clean/open/north

We assume that about directly applies the coarsest granularity function to its complement. This results in the same semantic effect as does approximately, though the mechanism is slightly different. We assume that approximately is not blocked by about because the resulting interpretation is identical.

$$
\llbracket \text { about } \mathrm{D} \rrbracket^{\text {gran }}=\operatorname{coarsest}(\operatorname{gran})(\llbracket \mathrm{D} \rrbracket)
$$

Some, weak disjunction, and approximative inversion in Russian are scalar approximators that can only be construed with numerals. (49a) illustrates the use of some we refer to, while (49b) illustrates what we call weak disjunction because the disjunction or must be unstressed for the approximator interpretation.

a. some fifteen boys

b. He is gone for two or three days.

In German, the equivalent of weak disjunction does not involve disjunction at all as illustrated in (50).

(50) Er ist für zwei drei Tage weg.

he is for two three days gone

Approximative inversion in Russian is illustrated by (51a). The postnominal position of the cardinal indicates that an approximative interpretation is intended.

a. Ja vstretil studentov desjat'.

I met students ten

'I met approximately 10 students'

b. Ja vstretil desjat' studentov

I met ten students

'I met ten students' 
We assume that all these approximators involve the same lexical entry as the one we gave for about. This is shown for approximative some in (52). For approximative inversion and weak disjunction we assume that the construction licenses a silent counterpart of approximative some.

$$
\llbracket \text { some } \mathrm{D} \rrbracket^{\text {gran }}=\operatorname{coarsest}(\text { gran }) \llbracket \mathrm{D} \rrbracket
$$

Partially has the same distribution as completely and more or less: As Kennedy and McNally (2005) have already observed, it can combine with predicates that introduce closed scales: ${ }^{13}$
a. partially dry/pure/empty
b. \#partially impure/three/north/white
c. \#partially Beef Stroganoff/a heap of wood

We perceive partially open to be equivalent to not completely closed. This follows from the lexical entry for partially in (54):

$$
\begin{aligned}
& \llbracket \text { partially } \rrbracket^{\text {gran }}=\lambda f \in D_{\langle d t, e t\rangle} \lambda x \in D_{e} \\
& \exists D \in D_{d t}[D=\text { finest }(\text { gran })(\min (\operatorname{domain}(f))) \& \neg f(D)(x)]
\end{aligned}
$$

\subsection{Further Potential Scalar Approximators}

The framework we develop for approximative modifiers calls for some discussion of almost, nearly, and barely. While we think that the analysis of these expressions also needs to make appeal to granularity, we do not have a fully worked out proposal to offer at this point. Briefly consider almost. It seems that almost does involve scales, but often the scale can be a derived temporal scale as the examples (55a) and (56a) illustrate (cf. Rapp and von Stechow 1996, Penka 2006). Approximately in (55b) and (56b) and the other scalar approximators we talked about above seem to be unable to access this scale.

a. John almost killed Harry. (with intended interpretation: John might have killed Harry.)

b. \#John approximately killed Harry.

a. Charles is almost King by now. (with intended interpretation: Charles will be King soon.)

b. \#Charles is approximately King by now.

In examples like (57), however, we can readily analyze almost as making reference to the interval one below the maximum on the coarsest granularity. To write out this lexical entry for almost in (57), we use the notation $\operatorname{prev}_{\text {gran }}(D)$ to denote the granularity interval on the scale of $D$ that precedes $D$.

\footnotetext{
${ }^{13}$ Manfred Bierwisch (p.c.) points out that the German counterpart of partially, 'teilweise', must always receive a mereological interpretation. A door that is 'teilweise' open, must be a door that consists out of at least two independently movable pieces, only one of which is open.
} 
(57)

The shirt is almost dry.

$$
\begin{aligned}
& \llbracket \text { aalmost } \rrbracket^{\text {gran }}=\lambda f \in D_{\langle d t, e t\rangle} \lambda x \in D_{e} \\
& \exists D \in D_{d t}\left[D=\operatorname{coarsest}(\operatorname{gran})(\max (\operatorname{domain}(f))) \& f\left(\operatorname{prev}_{\operatorname{gran}}(D)\right)(x)\right]
\end{aligned}
$$

The examples in (59) show that other scalar approximators can themselves be modified by almost. In this case, it seems that the resulting interpretation makes reference to the interval one below the maximum on the finest granularity.

The shirt was almost perfectly/completely dry.

\section{Conclusion}

In this paper, we argued for the necessity to distinguish between two kinds of vagueness, scalar and epistemic. We based this conclusion on a study of the semantic and distributional properties of approximators like exactly, completely, definitely, approximately, more or less, and maybe. One kind of vagueness relates precise expressions to intervals: We argued for a granularity parameter that regulates with which interval expressions like five o'clock that denote points on a scale are associated. Scalar approximators like exactly, completely, approximately, and more or less regulate the size of the granularity intervals. We argued in Section 4, that the scalar approximators are further subdivided into endpoint and non-endpoint oriented ones. The other kind of vagueness is genuinely epistemic, and epistemic expressions like definitely and maybe regulate this kind of vagueness.

One surprising result of our work is that heap and bald, two often cited examples of vague predicates, actually belong to two different classes of vague expressions: Heap is epistemically vague, while bald must be scalarly vague since it combines with the scalar approximators completely and more or less.

There are a number of things we had to put aside for reasons of space and time in this paper. This includes the analysis of the wider distribution not exactly, which as we noted is a phenomenon restricted to English, and of not even approximately. Furthermore, we did not actually provide an analysis of the approximator loosely speaking. This is one case that Lasersohn (1999) discusses, but his analysis is incompatible with our framework for the analysis of vagueness. Therefore, we are under an obligation to provide an alternative analysis, however, this is beyond the scope of the present paper for the reason that we believe that loosely speaking is speech-act oriented. One reason for this assumption is provided by the data in (60): Embedded under a speech-act verb in (60a), loosely speaking is part of the content of the embedded clause and interpreted from John's perspective. But, embedded under the non-speech-act verb think in $(60 \mathrm{~b})$, loosely speaking is interpreted from the speakers perspective and not part of the content of the embedded clause.

(60) a. John said that, loosely speaking, a heap of sand is on his table.

b. John thinks that, loosely speaking, a heap of sand is on his table. 


\section{References}

Barker, Chris: 2002, 'The dynamics of vagueness', Linguistics and Philosophy 25, $1-36$.

Bosch, Peter: 1983, “Vagueness" is context-dependence. A solution to the Sorites paradox', in T. T. Ballmer and M. Pinkal (eds.), Approaching Vagueness, 189-210. North Holland, Amsterdam, Netherlands.

von Fintel, Kai and Antony S. Gillies: 2006, 'Epistemic modality for dummies'. MIT and Michigan.

Heim, Irene and Angelika Kratzer: 1998, Semantics in Generative Grammar. Blackwell, Oxford, UK.

Hobbs, Jerry and Vladik Kreinovich: 2006, 'Optimal coice of granularity in commonsense estimation: Why half-orders of magnitude'. unpublished manuscript.

Kamp, Hans: 1981, 'The paradox of the heap', in U. Mönnich (ed.), Aspects of Philosophical Logic: Some Logical Forays into Central Notions of Linguistics and Philosophy, 225-277. Reidel, Dordrecht.

Kennedy, Christopher: 2007, 'Vagueness and grammar: the semantics of relative and absolute gradable adjectives', Linguistics and Philosophy 30, 1-45.

Kennedy, Christopher and Louise McNally: 2005, 'Scale structure and the semantic typology of gradable predicates', Language $\mathbf{8 1}$.

Krifka, Manfred: 2007, 'Approximate interpretation of number words: A case for strategic communication', in G. Bouma, I. Krämer, and J. Zwarts (eds.), Cognitive Foundations of Communication, 111-126. Koninklijke Nederlandse Akademie van Wetenschapen.

Lakoff, George: 1973, 'Hedges', Journal of Philosophical Logic 2, 458-508.

Lasersohn, Peter: 1999, 'Pragmatic halos', Language 75, 522-551.

Penka, Doris: 2006, 'Almost there: The meaning of almost', in Proceedings of SuB 10. Berlin.

Pinkal, Manfred: 1995, Logic and the Lexicon. Kluwer, Dordrecht, Netherlands.

Rapp, Irene and Arnim von Stechow: 1996, 'Fast 'almost' and the visibility parameter for D-adverbs', Journal of Semantics.

Rotstein, Carmen and Yoad Winter: 2004, 'Total adjectives vs. partial adjectives: Scale structure and higher-order modifiers', Natural Language Semantics 12, 259-288.

Schwarz, Bernhard: 2006, 'Modal superlatives', in Proceedings of SALT 15. CLC Publications, Cornell University, Ithaca, N.Y.

Siegel, Muffy: 2002, 'Like: The discourse particle and semantics', Journal of Semantics 19, 35-71.

Williamson, Timothy: 1994, Vagueness. Routledge, London, UK.

Williamson, Timothy: 2000, Knowledge and its Limits. Oxford University Press, Oxford, UK. 